\title{
PTOLEMAIC SPACES AND CAT(0)
}

\author{
S. M. BUCKLEY, K. FALK and D. J. WRAITH \\ Department of Mathematics, National University of Ireland Maynooth, Co. Kildare, Ireland \\ e-mail: stephen.m.buckley@gmail.com;kurt.falk@nuim.ie;david.wraith@nuim.ie
}

(Received 13 June 2007; revised 3 October 2008; accepted 15 October 2008)

\begin{abstract}
We consider Ptolemy's inequality in a metric space setting. It is not hard to see that $\mathrm{CAT}(0)$ spaces satisfy this inequality. Although the converse is not true in full generality, we show that if our Ptolemaic space is either a Riemannian or Finsler manifold, then it must also be CAT(0). Ptolemy's inequality is closely related to inversions of metric spaces. We exploit this link to establish a new characterization of Euclidean space amongst all Riemannian manifolds.
\end{abstract}

2000 Mathematics Subject Classification. 53C20, 53C60, 51F99.

1. Introduction. Inversion (or reflection) about the unit sphere is a bijection on $\mathbb{R}^{n} \backslash\{0\}$, so we can pull back Euclidean distance to get a new distance on $\mathbb{R}^{n} \backslash\{0\}$ : $i_{0}(x, y)=|x-y| /|x||y|$. Inversion has been generalized to the setting of a metric space $(X, d)$ in [7]: for fixed $p \in X$, define

$$
i_{p}(x, y)=\frac{d(x, y)}{d(x, p) d(y, p)}, \quad x, y \in X_{p}
$$

where $X_{p}:=X \backslash\{p\}$. In general this is not a metric on $X_{p}$, but a related function $d_{p}: X_{p} \times X_{p} \rightarrow[0, \infty)$ is defined and shown to be a metric which is subordinate to, but comparable with, $i_{p}$ (see [7, Lemma 3.2]).

The definition of $d_{p}$ is more complicated than that of $i_{p}$, so it is natural to ask when $i_{p}$ itself is a metric for all $p \in X$. This reduces to deciding if $i_{p}$ satisfies the triangle inequality, and is easily seen to be equivalent to Ptolemy's inequality

$$
d(x, y) d(z, p) \leq d(x, z) d(p, y)+d(x, p) d(y, z), \quad x, y, z, p \in X .
$$

A metric space satisfying this inequality is said to be Ptolemaic $[\mathbf{1}, \mathbf{4}, \mathbf{5}, \mathbf{9}, \mathbf{1 0}, \mathbf{1 3}, \mathbf{1 4}, \mathbf{1 5}]$.

It is not hard to see that CAT( 0 ) spaces are Ptolemaic (see Section 3 ). As a corollary, the inversions $i_{p}$ are metrics in every $\operatorname{CAT}(0)$ space. We also discuss implications in the converse direction in Section 3. The natural converse fails: there exists a geodesic Ptolemaic space that is not CAT(0) (see the comments after Theorem 1.1 in [9]). Nonetheless, if more structure is imposed on the space under consideration, it is possible to give converse statements. We prove the following result in Section 4.

Theorem 1.1. A complete Riemannian manifold $M$ is Ptolemaic if and only if it is $C A T(0)$, or equivalently a Hadamard manifold.

The first two authors were supported by Enterprise Ireland and Science Foundation Ireland. 
It has been brought to our attention that a local version of Theorem 1.1 was proven in the Ph.D. thesis of D. C. Kay. However, as that thesis is not easy to access, we include a complete proof of Theorem 1.1.

One might hope to get more examples of Ptolemaic spaces in the class of Finsler manifolds than in its subclass of Riemannian manifolds. The following result, proven in Section 5, says that this is not the case (see the thesis of D. C. Kay for a related result).

\section{Theorem 1.2. A Ptolemaic Finsler manifold is necessarily Riemannian.}

Finally, it seems natural to ask when the inversion metrics on a Riemannian manifold are themselves Riemannian. This question in fact allows us to characterize Euclidean space among all complete Riemannian manifolds, as revealed by the following result which is proved in Section 5. Recall that a Finsler manifold is a smooth manifold $M$ together with a function $F: T M \rightarrow[0, \infty)$ which is smooth on the complement of the zero section of $T M$, and which restricts on each tangent space to give a Minkowski norm. (A Minkowski norm is a norm which satisfies a certain strong convexity condition; see [2] for details.)

THEOREM 1.3. Let $M$ be a complete Riemannian manifold. Then the metric space $\left(M \backslash\{p\}, i_{p}\right)$ has the structure of a Riemannian manifold for all $p \in M$ if and only if $M$ is Euclidean space.

2. Preliminaries. A Riemannian manifold $M$ is a smooth manifold endowed with a Riemannian metric tensor $g$, that is, a choice of inner product on each tangent space $T_{x} M$ depending smoothly on $x \in M$.

A Finsler space is a smooth manifold $M$ endowed with a Finsler metric $F: T M \rightarrow$ $[0, \infty)$ which is $C^{\infty}$ on $T M \backslash\{0\}$ and whose restrictions $F_{q}=\left.F\right|_{T_{q} M}$ to the tangent spaces $T_{q} M, q \in M$, are Minkowski norms (for details see, for instance, [2]). The Finsler metric is introduced as in the Riemannian case: If $\gamma:[a, b] \rightarrow M$ is a Lipschitz continuous curve, then the function $t \mapsto F\left(\gamma^{\prime}(t)\right)$ is measurable and

$$
L_{F}(\gamma):=\int_{a}^{b} F\left(\gamma^{\prime}(t)\right) d t
$$

is the length of $\gamma$. The metric is then given by

$$
d(x, y):=\inf _{\gamma} L_{F}(\gamma), \quad x, y \in M,
$$

where the infimum is taken over all Lipschitz continuous curves $\gamma:[0,1] \rightarrow M$ with $\gamma(0)=x$ and $\gamma(1)=y$. Recall that the exponential map $\exp _{q}: T_{q} M \rightarrow M$ is defined as follows. Given $u \in T_{q} M$, $\exp _{q} u$ is the point in $M$ reached by a geodesic segment starting at $q$ in direction of $u$ which has length $F_{q}(u)$, assuming that such a geodesic movement is possible in $M$ (for further details see [2]).

In the rest of this paper, $(X, d)$ is a metric space and $X_{p}:=X \backslash\{p\} . X$ is said to be proper if all its closed balls are compact.

The one-point extension of $X$ is defined via

$$
\hat{X}:= \begin{cases}X & \text { when } X \text { is bounded, } \\ X \cup\{\infty\} & \text { when } X \text { is unbounded; }\end{cases}
$$


the open sets in $\hat{X}$ include those in $X$ together with complements (in $\hat{X}$ ) of closed balls (in $X$ ). Thus when $X$ is a proper space, $\hat{X}$ is simply its one-point compactification. Given a subspace $Z \subset X$, we write $\hat{Z}$ and $\hat{\partial} Z$ to denote the closure and boundary of $Z$ in $\hat{X}$; e.g. $\hat{Z}=\bar{Z}$ when $Z$ is bounded and $\hat{Z}=\bar{Z} \cup\{\infty\}$ when $Z$ is unbounded.

Recall the metric $d_{p}$ mentioned in Section 1 . When $(X, d)$ is unbounded, there is a unique point $p^{\prime}$ in the completion of $\left(X_{p}, d_{p}\right)$ which corresponds to the point $\infty$ in $\hat{X}$. (Any unbounded sequence in $\left(X_{p}, d\right)$ is a Cauchy sequence in $\left(X_{p}, d_{p}\right)$, and any two such sequences are equivalent.) Because of this phenomenon, we define

$$
\left(\operatorname{Inv}_{p}(X), d_{p}\right):=\left(\hat{X}_{p}, d_{p}\right)=\left(\hat{X} \backslash\{p\}, d_{p}\right)
$$

and we call $\left(\operatorname{Inv}_{p}(X), d_{p}\right)$ the inversion of $(X, d)$ with respect to the base point $p$. For example, with this definition, $\operatorname{Inv}_{p}(X)$ will be complete (or proper) whenever $X$ is complete (or proper).

Note that $i_{p}$ is a metric if and only if for all $x, y, z \in X_{p}$,

$$
i_{p}(x, y) \leq i_{p}(x, z)+i_{p}(z, y) .
$$

Writing $i_{p}(x, y)=d(x, y) / d(x, p) d(y, p)$ and similarly for the other two terms given above and then multiplying across by $d(x, p) d(y, p) d(z, p)$, we see that this last inequality becomes

$$
d(x, y) d(z, p) \leq d(x, z) d(p, y)+d(x, p) d(y, z), \quad x, y, z \in X_{p},
$$

so, as mentioned before, $i_{p}$ is a metric for all $p \in X$ if and only if $X$ is Ptolemaic.

A classical result says that Ptolemy's inequality holds in the Euclidean plane, with equality if and only if the points $x, y, z$ and $p$ lie on a circle in that order. In fact, this follows readily from the fact that $i_{p}$ is the pullback of Euclidean distance under inversion in the unit sphere. Ptolemy's inequality has also been studied in more general settings than Euclidean space (see for instance $[1,10,14]$ ). Of particular interest to us is the result of Schoenberg [15] which says that a normed space is Ptolemaic if and only if its norm is induced by an inner product.

We do not distinguish notationally between paths and their images. Suppose $\gamma$ : $I \rightarrow X$ is a path, where $I \subset \mathbb{R}$ is an interval. If $\gamma$ contains points $x, y \in X$, then $\gamma[x, y]$ denotes any subpath of $\gamma$ containing the points $x, y \in X$. This may not be unique: we insist only that different instances of such notation in the same proof refer to the same subpath.

If $(X, d)$ is rectifiably connected, we define the intrinsic metric associated with $d$ by

$$
l(x, y):=\inf \{\operatorname{len}(\gamma): \gamma \text { is a path in } X \text { containing } x, y\} .
$$

We say that $(X, d)$ is a length space if $l=d$. A path $\gamma$ of length $d(x, y)$ joining $x, y \in X$ is called a geodesic segment, and is often denoted by $[x, y]$. We call $(X, d)$ a geodesic space if all pairs of points can be joined by geodesic segments, i.e. the above infimum is always attained.

A geodesic triangle $T(x, y, z)$ is a collection of three points $x, y, z \in X$ together with a choice of geodesic segments $[x, y],[x, z]$ and $[y, z]$. Given such a geodesic triangle $\Delta:=T(x, y, z)$, a comparison triangle in this paper will always mean a geodesic triangle in the Euclidean plane, $T(\bar{x}, \bar{y}, \bar{z})$, such that corresponding distances coincide: $d(x, y)=$ $|\bar{x}-\bar{y}|, d(y, z)=|\bar{y}-\bar{z}|, d(z, x)=|\bar{z}-\bar{x}|$. Note that to avoid cluttered notation, we 
do not specify the space in the notation $T(\cdot, \cdot, \cdot)$; the space will always be clear from the context.

A triangle map (in $X$ ) is a function $f$ from a geodesic triangle $T(x, y, z)$ in $X$ onto a comparison triangle $T(\bar{x}, \bar{y}, \bar{z})$, such that $f(x)=\bar{x}, f(y)=\bar{y}, f(z)=\bar{z}$, and the restriction of $f$ to any one of the three sides is an isometry. Triangle maps always exist, and are unique up to an isometry of $\mathbb{R}^{2} .(X, d)$ is said to be a $C A T(0)$ space if it is geodesic and $d(u, v) \leq|f(u)-f(v)|$ for all $u, v \in \Delta$ whenever $f: \Delta \rightarrow \bar{\Delta}$ is a triangle map. A geodesic triangle is non-degenerate if each of its sides is strictly shorter than the sum of the lengths of the other two sides.

An important class of CAT( 0 ) spaces is given by Hadamard manifolds, i.e. complete, simply connected Riemannian manifolds of non-positive sectional curvature. Indeed a complete Riemannian manifold is CAT( 0$)$ if and only if it is a Hadamard manifold (see II.1.5, II.1A.6 and II.4.1 in [6]).

If $x, y, z$ are distinct points in $X$, then we define the angle $\theta:=\angle_{x}(y, z)$ by

$$
c^{2}=a^{2}+b^{2}-2 a b \cos \theta
$$

where $a:=d(x, y), b:=d(x, z)$ and $c:=d(y, z)$. Note that $\theta$ is the Euclidean angle at $\bar{x}$ whenever $T(\bar{x}, \bar{y}, \bar{z})$ is a comparison triangle for $T(x, y, z)$. If $\gamma:[0, a] \rightarrow X$ and $\gamma^{\prime}:[0, \bar{a}] \rightarrow X$ are geodesic paths, parametrized by arclength and with $\gamma(0)=\gamma^{\prime}(0)$, then we define the (Alexandrov) angle $\angle\left(\gamma, \gamma^{\prime}\right)$ by

$$
\angle\left(\gamma, \gamma^{\prime}\right):=\limsup _{t, t^{\prime} \rightarrow 0} \angle_{c(0)}\left(c(t), c\left(t^{\prime}\right)\right) .
$$

For much more on $\mathrm{CAT}(0)$ spaces and comparison angles, we refer the reader to [6]. Let us note that only Alexandrov angles correspond to the usual definition of angles in Euclidean and hyperbolic space; for the hyperbolic case see [6, I.2.9].

3. CAT(0) spaces are Ptolemaic. In this section, we discuss the following basic result:

\section{Proposition 3.1. Every CAT(0) space is Ptolemaic.}

This result follows easily from the fact (mentioned in [6, II.1.10]) that CAT(0) spaces $(X, d)$ satisfy the following $C A T(0)$ four-point condition:

For every choice of four points $x_{1}, x_{2}, y_{1}, y_{2} \in X$, there exist points $\bar{x}_{1}, \bar{x}_{2}, \bar{y}_{1}, \bar{y}_{2} \in$ $\mathbb{R}^{2}$ such that $d\left(x_{i}, y_{i}\right)=\left|\bar{x}_{i}-\bar{y}_{j}\right|, i, j=1,2$, and $d\left(x_{1}, x_{2}\right) \leq\left|\bar{x}_{1}-\bar{x}_{2}\right|$ and $d\left(y_{1}, y_{2}\right) \leq$ $\left|\bar{y}_{1}-\bar{y}_{2}\right|$. In fact, we simply take $\left(x_{1}, x_{1}, y_{1}, y_{2}\right)=(x, y, z, p)$ and use the fact that the Euclidean plane is Ptolemaic.

For the sake of completeness and since we shall later use some of the ideas contained in it, we also give the following direct proof:

$A$ direct proof of Proposition 3.1. Let $x, y, z$ and $p$ be arbitrary points in $X$. Consider Euclidean comparison triangles with vertices $\bar{x}, \bar{y}, \bar{z}$ and $\bar{x}, \bar{y}, \bar{p}$ corresponding to the triangles in $X$ with vertices $x, y, z$ and $x, y, p$, respectively. We choose the comparison triangles in such a way that $\bar{z}$ and $\bar{p}$ are not in the same open half-plane with respect to the line through $\bar{x}$ and $\bar{y}$.

We distinguish between two cases. In the first case, illustrated in Figure 1, the closed line segment with endpoints $\bar{z}$ and $\bar{p}$ intersects the closed line segment with endpoints $\bar{x}$ and $\bar{y}$. Let $\bar{u}$ be the intersection point and let $u$ be the corresponding 

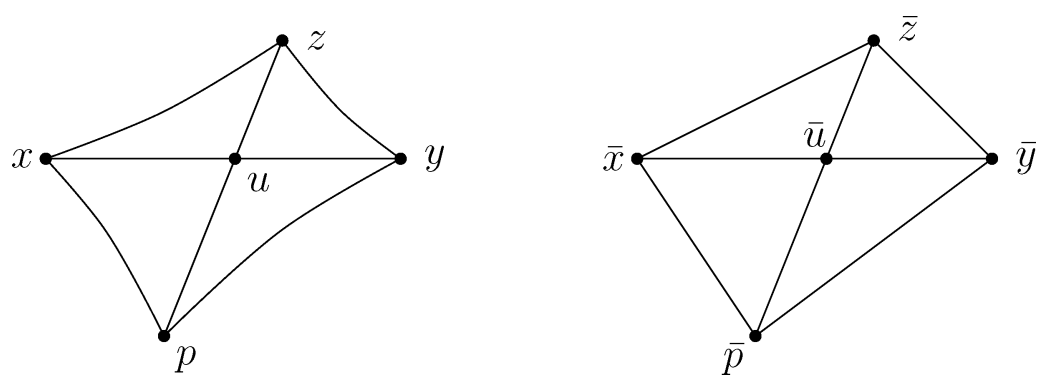

Figure 1 . The case where $[\bar{z}, \bar{p}]$ intersects $[\bar{x}, \bar{y}]$.

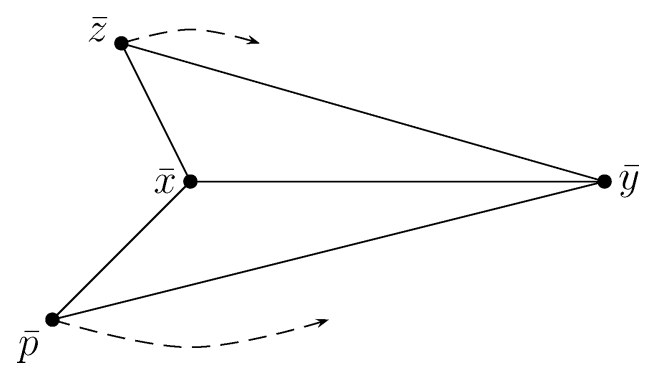

Figure 2. The non-intersecting case.

point on the geodesic segment connecting $x$ and $y$ in $X$, that is, the unique point $u$ on this geodesic segment such that $d(x, u)=|\bar{x}-\bar{u}|$. By the CAT(0) condition, we have $d(z, u) \leq|\bar{z}-\bar{u}|$ and $d(p, w) \leq|\bar{p}-\bar{u}|$, and so

$$
d(z, p) \leq d(z, u)+d(u, p) \leq|\bar{z}-\bar{u}|+|\bar{u}-\bar{p}|=|\bar{z}-\bar{p}| .
$$

It follows that Ptolemy's inequality holds for $d$, since all other distances involved are preserved, and the Euclidean plane is Ptolemaic.

We now consider the alternative Case 2 , when the closed segment with endpoints $\bar{z}$ and $\bar{p}$ does not intersect the closed segment from $\bar{x}$ to $\bar{y}$. In this case, we prove the stronger inequality

$$
d(x, y)(d(z, x)+d(x, p)) \leq d(x, z) d(y, p)+d(x, p) d(y, z),
$$

or equivalently

$$
|\bar{x}-\bar{y}|(|\bar{z}-\bar{x}|+|\bar{x}-\bar{p}|) \leq|\bar{x}-\bar{z}||\bar{y}-\bar{p}|+|\bar{x}-\bar{p}||\bar{y}-\bar{z}|,
$$

since the distances involved are all preserved by moving to the Euclidean plane.

To prove (4), we swivel the points $\bar{z}$ and $\bar{p}$ in such a way that the distances $|\bar{z}-\bar{x}|$ and $|\bar{x}-\bar{p}|$ are held constant and the angles $\angle_{\bar{x}}(\bar{z}, \bar{y})$ and $\angle_{\bar{x}}(\bar{p}, \bar{y})$ are decreasing. As long as the angles $\angle_{\bar{y}}(\bar{z}, \bar{x})$ and $\angle_{\bar{y}}(\bar{p}, \bar{x})$ are not allowed to exceed $\pi / 2$, swiveling in this manner decreases the distances $|\bar{z}-\bar{y}|$ and $|\bar{p}-\bar{y}|$ while holding the other three distances in (4) constant (see Figure 2). Thus the left-hand side of (4) is invariant under such a swivel and the right-hand side decreases, so (4) holds prior to such a swivel if it holds after the swivel.

A moment's reflection shows that we can always perform a swivel of this type to get $\angle_{\bar{x}}(\bar{z}, \bar{p})=\pi$ without allowing either $\angle_{\bar{y}}(\bar{z}, \bar{x})$ or $\angle_{\bar{y}}(\bar{p}, \bar{x})$ to exceed $\pi / 2$. But then 
we are back in Case 1, and (4) coincides with the usual Ptolemaic inequality because $|\bar{z}-\bar{x}|+|\bar{x}-\bar{p}|=|\bar{z}-\bar{p}|$. This concludes the proof of (4) in Case 2, and the proof of the proposition.

Let us note that (4) fails for certain configurations in Case 1. In fact if we take $\bar{x}, \bar{z}, \bar{y}, \bar{p}$ to be four distinct points lying on a Euclidean circle in that order, then Ptolemy's theorem tells us that

$$
|\bar{x}-\bar{y}||\bar{z}-\bar{p}|=|\bar{x}-\bar{z}||\bar{y}-\bar{p}|+|\bar{x}-\bar{p}||\bar{y}-\bar{z}| .
$$

Since $|\bar{z}-\bar{p}|<|\bar{z}-\bar{x}|+|\bar{x}-\bar{p}|$, we cannot have equality in (4).

A straightforward computation shows that Ptolemy's inequality (2) implies the triangle inequality for the inversion $i_{p}$ defined in (1), thus proving the following corollary of Proposition 3.1.

COROLlary 3.2. If $(X, d)$ is $C A T(0)$, then $i_{p}$ is a metric for all $p \in X$.

Let us now discuss possible converses to Proposition 3.1. Since a Ptolemaic space need not be geodesic (for instance the set of integers with the Euclidean metric inherits the Ptolemaic property from $\mathbb{R}$ ), one could not hope for all Ptolemaic spaces to be CAT(0). But it is not even true that Ptolemaic spaces can always be isometrically imbedded in a CAT(0) space. Consider for instance the space

$$
X=\{(0,0),(0,1),(1,1),(1,2)\}
$$

with the $l^{\infty}$ metric

$$
d\left(\left(x_{1}, y_{1}\right),\left(x_{2}, y_{2}\right)\right)=\max \left\{\left|x_{1}-y_{1}\right|,\left|x_{2}-y_{2}\right|\right\} .
$$

If $(X, d)$ is a subspace of a geodesic space $(Y, d)$, then the concatenation of geodesic segments $[(0,0),(0,1)]$ and $[(0,1),(1,2)]$ gives one geodesic segment from $(0,0)$ to $(1,2)$ while the concatenation of geodesic segments $[(0,0),(1,1)]$ and $[(1,1),(1,2)]$ gives another. The two segments have to be different since any segment from $(0,0)$ to $(1,2)$ that goes through both $(0,1)$ to $(1,1)$ must have length at least 3 . The $\operatorname{CAT}(0)$ condition readily implies uniqueness of geodesic segments so we are done.

A weaker converse implication would be the statement that a geodesic Ptolemaic space is $\operatorname{CAT}(0)$. This is not true in general (see [9]), but we prove it in the context of Riemannian manifolds in the next section.

4. Ptolemaic Riemannian manifolds are CAT(0). In this section we establish a converse to Proposition 3.1 in the Riemannian setting: a complete Riemannian manifold $(M, g)$ whose metric satisfies Ptolemy's inequality is necessarily CAT(0). In view of the fact that a CAT(0) manifold is precisely a Hadamard manifold, we must prove that a complete Ptolemaic manifold is simply connected (a global property) and is of non-positive sectional curvature (a local property). It makes sense to separate these two implications. We begin with the global one, which we prove in a more general context than manifolds.

THeOREM 4.1. If a Ptolemaic length space is complete and semilocally simply connected, then it must be simply connected. 
Before giving the proof, let us explain the idea. First note that a circle of perimeter $4 L$ with the arclength metric attached is not Ptolemaic. Indeed, if we choose the points $x, z, y, p$ to be quarter points on the circle, i.e. four points in this order around the circle that divide the circle into four pieces of equal length, then

$$
d(x, y) d(z, p)=(2 L)(2 L)=4 L^{2}>L^{2}+L^{2}=d(x, z) d(y, p)+d(x, p) d(y, z) .
$$

Thus, any metric space $X$ containing an isometric copy of a circle is non-Ptolemaic. A space that fails to be simply connected might fail to have an isometric copy of a circle, but Ptolemy's inequality still fails if we can find a set of four points in $X$ for which the set of distances between pairs approximates sufficiently well the distances between quarter points of a circle. The proof of Theorem 4.1 amounts to finding a set of such approximate quarter points.

Recall that a topological space $X$ is semilocally simply connected if each point $x \in X$ has a neighbourhood $U$ such that each loop at $x$ contained in $U$ is contractible within $X$.

Proof of Theorem 4.1. We assume that $(X, d)$ is a complete, semilocally simply connected length space which fails to be simply connected. To prove the theorem, we are required to prove that $X$ cannot be Ptolemaic. The proof is a stopping time construction involving non-contractible loops $\gamma_{j}$ at basepoints $p_{j}$, for $j \leq j_{0}$ where $j_{0} \in \mathbb{N} \cup\{\infty\}$ is the stopping index.

We begin by picking any basepoint $p_{1} \in X$ and a non-contractible loop $\gamma_{1}$ : $\left[0,4 L_{1}\right] \rightarrow X$ parametrized by arclength with $\gamma_{1}(0)=\gamma_{1}\left(4 L_{1}\right)=p_{1}$ and the following near-minimality property: $4 L_{1}<(7 / 6) \operatorname{len}(\gamma)$ for all non-contractible loops $\gamma$ at $p_{1}$ (see Figure 3). Note that semilocal simple connectedness guarantees that this is possible. By writing $l_{1}:=\gamma_{1}\left(L_{1}\right), u_{1}:=\gamma_{1}\left(2 L_{1}\right), r_{1}:=\gamma_{1}\left(3 L_{1}\right)$, we divide the loop into four subpaths of length $L_{1}$.

It is convenient to define the left half and right half of $\gamma_{1}$ to be the paths from $p_{1}$ to $u_{1}$ given by the joins $\gamma_{1}\left[p_{1}, l_{1}\right]+\gamma_{1}\left[l_{1}, u_{1}\right]$ and $\gamma_{1}\left[u_{1}, r_{1}\right]+\gamma_{1}\left[r_{1}, p_{1}\right]$, respectively. Similarly, we define the upper and lower halves of $\gamma_{1}$ to be the paths from $l_{1}$ to $r_{1}$ obtained by joining the two of these four subarcs of $\gamma_{1}$ that have $u_{1}$ or $p_{1}$ as an endpoint, respectively.

We claim that $d\left(p_{1}, u_{1}\right) \geq 10 L_{1} / 7$. Suppose for the sake of contradiction that this is not true, and so we can find a path $\lambda_{1}$ from $p_{1}$ to $u_{1}$ of length less than $10 L_{1} / 7$. Joining $\lambda_{1}$ with either the left or right half of $\gamma$, we get two loops at $p_{1}$. Since $\gamma_{1}$ is non-contractible, at least one of these two new loops is non-contractible. But both have length less than $2 L_{1}+10 L_{1} / 7=(6 / 7)\left(4 L_{1}\right)$ and this contradicts the assumed near-minimality of $\gamma_{1}$. Thus the claim follows.

The stopping condition for this first step is $d\left(l_{1}, r_{1}\right) \geq 10 L_{1} / 7$. Since the distances $d\left(p_{1}, l_{1}\right), d\left(l_{1}, u_{1}\right), d\left(u_{1}, r_{1}\right)$ and $d\left(r_{1}, p_{1}\right)$ are all at most $L_{1}$, and $d\left(p_{1}, u_{1}\right) \geq 10 L_{1} / 7$, it follows that if the stopping condition holds, then

$$
d\left(l_{1}, r_{1}\right) d\left(p_{1}, u_{1}\right) \geq\left(\frac{10 L_{1}}{7}\right)^{2}>L_{1}^{2}+L_{1}^{2} \geq d\left(l_{1}, p_{1}\right) d\left(r_{1}, u_{1}\right)+d\left(l_{1}, u_{1}\right) d\left(r_{1}, p_{1}\right),
$$

and so the space is not Ptolemaic, as required.

Suppose instead that the stopping condition fails, so that there exists a path $v_{1}$ from $l_{1}$ to $r_{1}$ of length less than $10 L_{1} / 7$. Joining $v_{1}$ with either the upper or lower half of $\gamma_{1}$, we get two new loops which we term the upper and lower loops, respectively. The non-contractibility of the lower loop contradicts the assumed near-minimality of 


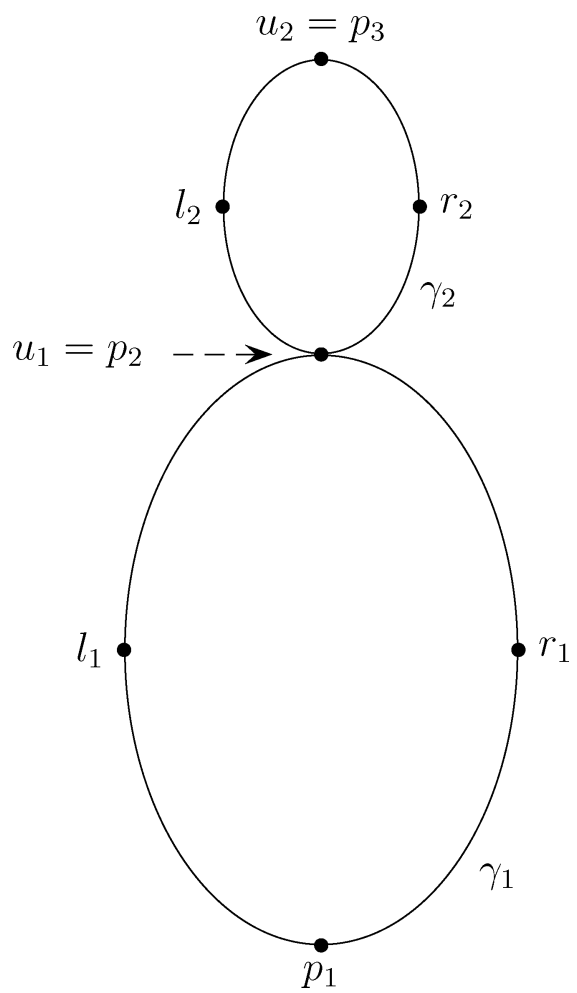

Figure 3. The first two steps of the construction.

$\gamma_{1}$ as before, so we may assume that it is contractible and thus that the upper loop is non-contractible. Note that the non-contractibility of the upper loop does not lead to a contradiction because this loop does not pass through $p_{1}$.

We define $p_{2}=u_{1}$. Since the upper loop is non-contractible and has length less than $(6 / 7) 4 L_{1}$, semilocal simple connectedness implies that we can find a non-contractible loop $\gamma_{2}:\left[0,4 L_{2}\right] \rightarrow X$, parametrized by arclength, with $\gamma_{2}(0)=\gamma_{2}\left(4 L_{2}\right)=p_{2}, L_{2}<$ $(6 / 7) L_{1}$, and the following near-minimality property: $4 L_{2}<(7 / 6) \operatorname{len}(\gamma)$ for all noncontractible loops $\gamma$ at $p_{2}$.

The construction proceeds in the obvious inductive manner. If at any stage the corresponding stopping condition applies, then the space is not Ptolemaic. To finish the proof, we will show that failure of the stopping condition at every step leads to a contradiction. Indeed this is easy: since the paths $\gamma_{j}$ have length

$$
L_{j}<(6 / 7)^{j-1} L_{1} \rightarrow 0 \quad \text { as } j \rightarrow \infty
$$

the sequence $\left(p_{j}\right)$ necessarily converges to some point $p \in X$ and, for sufficiently large $j$, the non-contractible path $\gamma_{j}$ lies in an arbitrarily small neighbourhood of $p$, contradicting semilocal simple connectedness.

Let us remark that it is proved in [9, Theorem 1.2] that a proper Ptolemaic space is uniquely geodesic and so contractible. In particular it is simply connected. However 
properness is a rather strong condition, not satisfied by infinite-dimensional normed spaces, for instance.

It remains to prove that a Ptolemaic Riemannian manifold has non-positive curvature.

THEOREM 4.2. If a Riemannian manifold $(M, g)$ of dimension $n \geq 2$ is Ptolemaic, then it has non-positive sectional curvature.

The proof of Theorem 4.2 requires a strict Topogonov type result comparing hinges in spaces of constant curvature, given in the following lemma, and a consequence of this lemma.

In the following two results, a hinge simply means a triple of points in a metric space. A pair of hinges $\{u, v, w\}$ and $\{\bar{u}, \bar{v}, \bar{w}\}$ (in typically different metric spaces both of whose metrics we denote by $d$ ) are said to be comparison hinges if $d(u, v)=d(\bar{u}, \bar{v})$, $d(u, w)=d(\bar{u}, \bar{w})$ and $\angle_{u}(v, w)=\angle_{\bar{u}}(\bar{v}, \bar{w})$. Note that the major difference between this concept and that of comparison triangles is that we require two distances and an angle to match rather than three distances.

LEMMA 4.3. Suppose we have comparison hinges $\left\{v, p_{1}, p_{2}\right\}$ in a space form of constant curvature $\delta \neq 0$ and $\left\{\bar{v}, \overline{p_{1}}, \overline{p_{2}}\right\}$ in $\mathbb{R}^{n}$. If the angle $\alpha=\angle_{v}\left(p_{1}, p_{2}\right)=\angle_{\bar{v}}\left(\overline{p_{1}}, \overline{p_{2}}\right)$ lies in $(0, \pi)$, then

(i) $d\left(p_{1}, p_{2}\right)<\left|\overline{p_{1}}-\overline{p_{2}}\right|$ if $\delta>0$;

(ii) $d\left(p_{1}, p_{2}\right)>\left|\overline{p_{1}}-\overline{p_{2}}\right|$ if $\delta<0$.

Proof. Given the hinge in $\mathbb{R}^{n}$, we can assume that the comparison hinge in the space form $M$ arises in the following way. Choose any point $v \in M$, and identify the origin of the tangent space to $M$ at this point with $\bar{v}$. Corresponding to the points $\overline{p_{1}}$, $\overline{p_{2}} \in T_{v} M$, we set $p_{i}=\exp \left(\bar{p}_{i}\right)$ for $i=1,2$. This gives the desired hinge in $M$.

We focus first on the case $\delta>0$. Consider the geodesic $\gamma(t)$ in $T_{v} M$ joining $\overline{p_{1}}$ to $\overline{p_{2}}$. As $\alpha \neq 0, \pi$, for each $t$ we have a non-zero component of $\gamma^{\prime}(t)$ orthogonal to the line from $\bar{v}$ to $\gamma(t)$. Since $|\bar{v}-\gamma(t)|>0$, it follows easily from a result of Rauch [3, Proposition 74] that each of these orthogonal components is strictly longer than the image of the component under the (derivative of the) exponential map at $\bar{v}$. Since $\exp _{*}$ preserves the length of vectors along any geodesic from $\bar{v}$, we see that

$$
\left|\exp _{*} \gamma^{\prime}(t)\right|<\left|\gamma^{\prime}(t)\right| \text {. }
$$

For future reference, observe that if $\delta<0$, this inequality would be reversed. The path $\exp \gamma(t)$ joining $p_{1}$ to $p_{2}$ is therefore strictly shorter than $\gamma(t)$. But the path $\exp \gamma(t)$ has length at least as long as the distance in $M$ between $p_{1}$ and $p_{2}$. Hence $d\left(p_{1}, p_{2}\right)<\left|\overline{p_{1}}-\overline{p_{2}}\right|$, as claimed.

We now consider the case $\delta<0$. This time let $\gamma(t)$ be a minimal geodesic joining $p_{1}$ to $p_{2}$ in $M$. By the arguments used above, we see that the path $\exp ^{-1} \gamma(t)$ joining $\overline{p_{1}}$ to $\overline{p_{2}}$ in $T_{v} M$ has strictly shorter length than $\gamma(t)$, since the velocity vectors to $\exp ^{-1} \gamma(t)$ must have some component orthogonal to the radial direction from $\bar{v}$, at least for $t$ in some open interval. Moreover, this path in $T_{v} M$ is at least as long as the Euclidean distance between $\overline{p_{1}}$ and $\overline{p_{2}}$. Hence $d\left(p_{1}, p_{2}\right)>\left|\overline{p_{1}}-\overline{p_{2}}\right|$, as required.

Corollary 4.4. Consider a hinge $\left\{w, q_{1}, q_{2}\right\}$ in a manifold $M$ of curvature $K>0$, with angle $\angle_{w}\left(q_{1}, q_{2}\right) \in(0, \pi)$, and let $\left\{\bar{v}, \overline{p_{1}}, \overline{p_{2}}\right\}$ be a comparison hinge in Euclidean space. Then $d_{M}\left(q_{1}, q_{2}\right)<\left|\overline{p_{1}}-\overline{p_{2}}\right|$. 
Proof. The hinge $\left\{w, q_{1}, q_{2}\right\}$ lies inside some compact region of the manifold. Over this region, the curvature is bounded away from 0 , that is, $K \geq \delta>0$. Let $\left\{v, p_{1}, p_{2}\right\}$ be a comparison hinge in the space form $X$ of constant curvature $\delta$. By the Toponogov Theorem, we have $d_{M}\left(q_{1}, q_{2}\right) \leq d_{X}\left(p_{1}, p_{2}\right)$. By Lemma 4.3, we also have $d_{X}\left(p_{1}, p_{2}\right)<$ $\left|\overline{p_{1}}-\overline{p_{2}}\right|$. Thus $d_{M}\left(q_{1}, q_{2}\right)<\left|\overline{p_{1}}-\overline{p_{2}}\right|$, as claimed.

Proof of Theorem 4.2. If the curvature of $M$ fails to be non-positive, we can find $p \in M$ and a two-dimensional subspace $V$ of $T_{p} M$ such that the sectional curvature $K(V)>0$.

Restricting the exponential map at $p$ to $V$, we obtain a local diffeomorphism from a neighbourhood of the origin in $V$ onto a two-dimensional submanifold of $M$. By openness of the positivity condition, the Gaussian curvature of this submanifold will be strictly positive close to $p$. In particular there exists $\epsilon>0$ such that the closed $\epsilon$ ball centred at $p$ in our submanifold consists entirely of points with positive Gaussian curvature. Call this ball $B$.

Now choose linearly independent vectors $v_{1}, v_{2}$ in $V$ of equal length less than $\epsilon$. Set $v_{-i}=-v_{i}$ for $i=1,2$; set $x_{i}=\exp \left(v_{i}\right)$ for $i=-2,-1,1,2$. Let $d_{B}$ and $d_{M}$ respectively denote distances measured in $B$ and $M$, and let $d$ denote distances in $V$. Clearly $d_{B}\left(x_{i}, x_{j}\right) \geq d_{M}\left(x_{i}, x_{j}\right)$ in general, and $d_{B}\left(x_{i}, x_{-i}\right)=d_{M}\left(x_{i}, x_{-i}\right)=d\left(v_{i}, v_{-i}\right)$. Linear independence means that for $|i| \neq|j|$, the angle $\angle_{0}\left(v_{i}, v_{j}\right)=\angle_{p}\left(x_{i}, x_{j}\right) \in(0, \pi)$, and since the curvature is strictly positive, Corollary 4.4 applies. From this we deduce that for such $i, j$,

$$
d_{M}\left(x_{i}, x_{j}\right) \leq d_{B}\left(x_{i}, x_{j}\right)<d\left(v_{i}, v_{j}\right) .
$$

Since the $\left\{v_{i}\right\}$ lie on a Euclidean circle, the Ptolemy inequality is in fact an equality for these points. However, the above estimates show that the Ptolemy inequality does not hold in $M$ for the points $\left\{x_{i}\right\}$, so $M$ cannot be a Ptolemaic space.

5. Ptolemaic Finsler spaces are Riemannian. In view of the aforementioned result of Schoenberg [15] that normed spaces are Ptolemaic if and only if their norm is induced by an inner product, one can also consider the question of finding a converse to Proposition 3.1 in the setting of Finsler geometry. As sated in Theorem 1.2, it turns out that if the Finsler metric of a Finsler space satisfies Ptolemy's inequality, then it is in fact Riemannian. The proof uses Schoenberg's result [15] and a theorem of Whitehead asserting that the differential at the origin of the exponential map of a Finsler space is always the identity $[\mathbf{2}, \mathbf{1 6}]$.

Proof of Theorem 1.2. There is a concept of exponential map for Finsler manifolds, and this map exists at least in some neighbourhood of each point (see [2, p. 126]). Moreover, it is $C^{1}$ at the zero-section of $T M$, and smooth away from the zero-section [2, p. 126-127]. A theorem of Whitehead states that the derivative of the exponential map at its origin is always the identity $[\mathbf{2}, \mathbf{1 6}]$.

Given any point $p$ in our Finsler manifold $\left(M^{n} ; F\right)$, the existence of a pointed differentiable map $\left(T_{p} M, 0\right) \rightarrow(M, p)$ which is isometric at 0 , such as the exponential map, is sufficient to ensure the pointed Gromov-Hausdorff convergence of the scaled spaces $(M, p ; \lambda F)$ to the space $\left(T_{p} M, 0 ; F_{p}\right)$ as $\lambda \rightarrow \infty$. Here, $F_{p}$ denotes the Minkowski norm on the tangent space $T_{p} M$. (See for example Proposition 3.15 of [12]. This deals with the Riemannian analogue of the above, but the same argument is easily seen to work in the Finsler case.) 
The Ptolemaic condition is preserved both by scaling and under GromovHausdorff convergence. Its preservation by scaling is trivial. To see why it is preserved under Gromov-Hausdorff convergence, consider a sequence of Ptolemaic spaces $X_{i}$ converging to a metric space $X$. If $X$ is not Ptolemaic, then we can find four points $x, y, z, p$ such that

$$
d(x, y) d(z, p)>d(x, z) d(y, p)+d(x, p) d(y, z) .
$$

Since this inequality is strict, there is an $\epsilon>0$ such that the inequality

$$
(d(x, y)-\epsilon)(d(z, p)-\epsilon)>(d(x, z)+\epsilon)(d(y, p)+\epsilon)+(d(x, p)+\epsilon)(d(y, z)+\epsilon)
$$

holds. For this $\epsilon$ and for $i$ sufficiently large, we can find $\epsilon$-Hausdorff approximations $X_{i} \rightarrow X$ and $X \rightarrow X_{i}$ (see [11] p. 146 for details). It follows from the second inequality that the images in $X_{i}$ of $x, y, z, p$ under such a map must also fail to satisfy the Ptolemy inequality. Therefore, since each $X_{i}$ is assumed Ptolemaic we deduce that we cannot find four points in $X$ for which the Ptolemy condition fails. In other words, $X$ must be Ptolemaic.

Given that our Finsler manifold is Ptolemaic, we now deduce that the tangent cone at $p,\left(T_{p} M ; F_{p}\right)$ is also Ptolemaic. By a result of Schoenberg [15], this means that the norm $F_{p}$ is induced by an inner product. Since this is true for all $p \in M$, we see that $M$ is actually a Riemannian manifold, as claimed.

Summarizing our results for manifolds so far, we have in effect proven the following equivalence.

THEOREM 5.1. For a smooth manifold $M$ the following conditions are equivalent:

(i) $M$ is Finsler and Ptolemaic.

(ii) $M$ is Finsler and $C A T(0)$.

(iii) $M$ is Hadamard.

Proof. The implication (ii) $\Rightarrow$ (i) follows from Proposition 3.1 which states that Ptolemy's inequality is satisfied in any geodesic CAT(0)-space. Note that this result holds in more generality than the Finsler manifolds we consider here.

In order to prove (i) $\Rightarrow$ (iii) we first observe that by Theorem 1.2 the fact that Ptolemy's inequality is satisfied in the Finsler manifold $M$ implies that $M$ must be Riemannian. Furthermore, Theorem 4.1 ensures that $M$ must be simply connected. Finally, if $M$ is assumed not to have non-positive sectional curvature, it follows by Theorem 4.2 that Ptolemy's inequality fails. Therefore, $M$ must have non-positive curvature, and thus is Hadamard.

The implication (iii) $\Rightarrow$ (ii) is trivial since $M$ is assumed to be Hadamard and is thus Finsler, and since any simply connected Riemannian manifold with non-positive curvature is $\operatorname{CAT}(0)$.

6. When are the inversions of a manifold Riemannian? In this section, we prove Theorem 1.3. In fact we prove more, as revealed by the following result. (For the statement, recall that the only difference between $\left(M_{p}, i_{p}\right)$ and $\operatorname{Inv}_{p}(M)$ is that the latter space includes a point at infinity if $M$ is unbounded.)

THEOREM 6.1. Let $M$ be a complete Riemannian manifold of dimension $n \geq 1$. The following are equivalent: 
(a) $M$ is Euclidean n-space;

(b) $\operatorname{Inv}_{p}(M)$ has the structure of a Riemannian manifold, for all $p \in M$;

(c) $\left(M_{p}, i_{p}\right)$ has the structure of a Riemannian manifold, for all $p \in M$;

(d) $\operatorname{Inv}_{p}(M)$ is a length space, for all $p \in M$;

(e) $\left(M_{p}, i_{p}\right)$ is a length space, for all $p \in M(n>1$ only).

Proof. Most of the implications of this theorem are easy, so let us deal with the easy cases first. If $M$ is Euclidean $n$-space, then we know that $\left(M_{p}, i_{p}\right)$ is isometric to Euclidean $n$-space with one point removed and that $\operatorname{Inv}_{p}(M)$ is isometric to Euclidean $n$-space, so it is immediate that (a) implies (b), (c), (d) and (e). Trivially (b) implies (c) and (d), both of which in turn imply (e). Moreover the case $n=1$ is easy since the only complete Riemannian manifolds are $M=\mathbb{R}^{1}$ (in which case $\operatorname{Inv}_{p}(M)$ is isometric to the Euclidean line and $\left(M_{p}, i_{p}\right)$ is isometric to the Euclidean line with a point removed), and $M=\mathbb{S}^{1}$ (in which case $i_{p}$ is not a metric by Theorem 4.1). Therefore it suffices to prove that (e) $\Rightarrow$ (a), and we may assume that $n>1$.

Note that by Theorem 1.1, (e) already implies that $M$ is a Hadamard manifold. So all we need to show is that if the sectional curvature of $M$ is non-zero at some point $p \in M$, then $i_{p}$ is not a length metric. This follows from a more general statement proven in the next proposition.

Proposition 6.2. Let $X$ be a CAT(0) space and $p \in X$ such that $i_{p}$ is a geodesic metric. Then, for all $x, y \in X$ such that $x, y, p$ do not lie on a common geodesic, the triangle with vertices $x, p, y$ is flat. Thus, $X$ is isometric to a convex subset of a Euclidean cone with vertex $p$.

Proof. Since $x, y, p$ do not lie on a common geodesic, the geodesic triangle $\Delta:=$ $T(x, y, p)$ is non-degenerate. Suppose that there exists an $i_{p}$-geodesic segment $\gamma$ in $\operatorname{Inv}_{p}(M)$ for the pair $x, y$. In $(X, d), \gamma$ is either a single path between $x$ and $y$ (if $p^{\prime} \notin \gamma$ ), or a pair of paths from $x$ and $y$ to infinity (if $p^{\prime} \in \gamma$ ). In either case, we can pick a point $z \in \gamma$, with $0<d(x, z)<d(x, y) / 2$. Now $i_{p}(x, y)=i_{p}(x, z)+i_{p}(z, y)$, and it follows easily that

$$
d(x, y) d(z, p)=d(x, z) d(p, y)+d(x, p) d(y, z) .
$$

We thus have equality in the Ptolemy inequality for the points $p, x, y, z$.

Recall that the subembedding theorem [6, II.1.10] states that CAT $(0)$ spaces $(X, d)$ satisfy the CAT(0) four-point condition: For every choice of four points $x_{1}, x_{2}, y_{1}, y_{2} \in$ $X$, there exist points $\bar{x}_{1}, \bar{x}_{2}, \bar{y}_{1}, \bar{y}_{2} \in \mathbb{R}^{2}$ such that $d\left(x_{i}, y_{i}\right)=\left|\bar{x}_{i}-\bar{y}_{j}\right|, i, j=1,2$, and $d\left(x_{1}, x_{2}\right) \leq\left|\bar{x}_{1}-\bar{x}_{2}\right|$ and $d\left(y_{1}, y_{2}\right) \leq\left|\bar{y}_{1}-\bar{y}_{2}\right|$. The rigidity part of the subembedding theorem states that if there are four points $x_{1}, x_{2}, y_{1}, y_{2}$ in a CAT(0) space $X$ such that the above equalities hold, and the inequalities become equalities, then the convex hull of $x_{1}, x_{2}, y_{1}, y_{2}$ in $X$ is isometric to the corresponding Euclidean comparison quadrangle. This statement can be proven along the following lines. Assume for simplicity that the points $\bar{x}_{1}, \bar{y}_{1}, \bar{x}_{2}, \bar{y}_{2}$ form a convex quadrilateral in $\mathbb{R}^{2}$ and let $\bar{m}$ be the intersection point of the diagonals $\left[\bar{x}_{1}, \bar{x}_{2}\right]$ and $\left[\bar{y}_{1}, \bar{y}_{2}\right]$. Furthermore, let $m_{1}$ and $m_{2}$ be the comparison points corresponding to $\bar{m}$ on the geodesic segments with endpoints $x_{1}, x_{2}$ and $y_{1}, y_{2}$, respectively. By using the CAT( 0 ) property and the triangle inequality one can readily show that $m_{1}$ and $m_{2}$ coincide. We can then apply [8, Proposition 9.1.19] to obtain that all triangles $T\left(x_{1}, y_{1}, x_{2}\right), T\left(x_{2}, y_{2}, x_{1}\right), T\left(x_{1}, y_{1}, y_{2}\right)$ and $T\left(y_{1}, x_{2}, y_{2}\right)$ are flat. 
Now set $x_{1}=x, y_{1}=p, x_{2}=y, y_{2}=z$ and assume that either one or both the inequalities in the four-point condition is a strict inequality. Then, by 5 ,

$$
\begin{aligned}
|\bar{x}-\bar{y}||\bar{z}-\bar{p}| & >d(x, y) d(z, p) \\
& =d(x, z) d(p, y)+d(x, p) d(y, z) \\
& =|\bar{x}-\bar{z}||\bar{p}-\bar{y}|+|\bar{x}-\bar{p}||\bar{y}-\bar{z}|
\end{aligned}
$$

which contradicts the Ptolemaic inequality in $\mathbb{R}^{2}$. Thus, we can apply the rigidity part of the subembedding theorem and obtain in particular that the triangle $\Delta$ is flat. This finishes the proof.

REMARK 6.3. We claim that if $x, y$ are points in $M \backslash\{p\}$ and there is an $i_{p}$-geodesic in $\operatorname{Inv}_{p}(M)$ from $x$ to $y$, then $x, y$ and $p$ necessarily lie on a common geodesic segment.

We prove (the contrapositive of) our claim. Suppose $x, y, p$ do not lie on a common geodesic. As in the proof of Proposition 6.2, consider the non-degenerate geodesic triangle $\Delta=T(x, y, p)$. We can again pick a point $z$ such that (5) holds.

Now, examining the proof of Proposition 3.1, we see that in the intersecting case, we can only have (5) if the corresponding equation also holds in the Euclidean comparison picture. By Ptolemy's theorem, this equation can only hold when the four points $\bar{x}, \bar{y}$, $\bar{z}$ and $\bar{p}$ all lie on a common line or circle. The four points cannot lie on a common line because $T(x, y, p)$, and so also $T(\bar{x}, \bar{y}, \bar{p})$, is non-degenerate. Therefore we can assume that they lie on a common circle. This rules out the possibility that $\bar{z}, \bar{p}$, and either $\bar{x}$ or $\bar{y}$ lie on a common line. Thus the point $\bar{u}$ (mentioned in the proof for the intersecting case) cannot be $\bar{x}$ or $\bar{y}$, and so $u \neq x, y$. We can therefore apply Lemma 4.3 to deduce that $d(u, p)<|\bar{u}-\bar{p}|$. This strict inequality leads to a strict version of (3), and so we cannot have equality in Ptolemy's inequality.

In the non-intersecting case, we got the stronger inequality

$$
d(x, y)(d(z, x)+d(x, p)) \leq d(x, z) d(y, p)+d(x, p) d(y, z) .
$$

When we swivel to reduce to Case 1, as described in the proof of Proposition 3.1, the right-hand side gets strictly smaller and the left-hand side remains unchanged, so (5) must fail in this case also.

In view of the above claim, the question naturally arises as to what happens when $x, y, p$ lie on a common geodesic. Suppose that $x \in[p, y], x \neq p, y$. According to (4.2) in [7], the length $\ell_{p}(\gamma)$ of a path $\gamma$ with respect to $i_{p}$ is given by

$$
\ell_{p}(\gamma)=\int_{\gamma} \frac{|d x|}{d(x, p)^{2}}
$$

where $|d x|$ is the length element for $(X, d)$. Thus

$$
\ell_{p}([x, y])=\int_{d(x, p)}^{d(y, p)} \frac{d t}{t^{2}}=\frac{1}{d(x, p)}-\frac{1}{d(y, p)}=\frac{d(y, p)-d(x, p)}{d(x, p) d(x, p)}=i_{p}(x, y) .
$$

Using a similar analysis to the proof of Theorem 6.1 , the reader can check that $l_{p}(x, y)>i_{p}(x, y)$ if $p \in[x, y], p \neq x, y$.

ACKNOWLEDGEMENTS. The authors wish to thank Jane McDougall for her careful reading of this paper and for useful discussions. The authors are also indebted to 
the referee whose suggestions and simplifications of proofs have been very useful in producing the final version of the manuscript. The first two authors were supported by Enterprise Ireland and Science Foundation Ireland.

\section{REFERENCES}

1. V. V. Aseev, A. S. Sychëv and A. V. Tetenov, Möbius-invariant metrics and generalized angles in Ptolemaic spaces, Siberian Math. J. 46 (2005) 189-204.

2. D. Bao, S. S. Chern and Z. Shen, An introduction to Riemann-Finsler geometry (Springer Verlag, New York, 2000).

3. M. Berger, A panoramic view of riemannian geometry (Springer Verlag, Berlin, Heidelberg, New York, 2003).

4. L. M. Blumenthal, Remarks on a weak four-point property, Revista Ci. Lima 45 (1943), $183-193$

5. L. M. Blumenthal, Theory and applications of distance geometry, 2nd Ed. (Chelsea Publishing Co., New York, 1970).

6. M. R. Bridson and A. Haefliger, Metric spaces of non-positive curvature (Springer Verlag, Berlin, 1999).

7. S. M. Buckley, D. Herron and X. Xie, Metric space inversions, quasihyperbolic distance, and uniform spaces, Preprint (2006).

8. D. Burago, Y. Burago and S. Ivanov, A course in metric geometry, in Graduate studies in mathematics, vol. 33 (AMS, 2001).

9. T. Foertsch, A. Lytchak and V. Schroeder, Nonpositive curvature and the Ptolemy inequality, Int. Math. Res. Not. 2007 (2007), article ID rnm100.

10. T. Foertsch and V. Schroeder, Hyperbolicity, CAT(-1)-spaces and the Ptolemy inequality, Preprint (2006).

11. K. Fukaya, Hausdorff convergence of Riemannian manifolds and its applications, $A d v$. Stud. Pure Math. 18-I (1990), 143-238.

12. M. Gromov, Metric structures for Riemannian and non-Riemannian spaces, in Progress in Mathematics vol. 152 (Birkhäuser, 2001).

14. M. S. Klamkin and A. Meir, Ptolemy's inequality, chordal metric, multiplicative metric, Pacific J. Math. 101 (1982) 389-392.

15. I. J. Schoenberg, A remark on M. M. Day's characterization of inner-product spaces and a conjecture of L. M. Blumenthal, Proc. Amer. Math. Soc. 3 (1952) 961-964.

16. I. J. Whitehead, Convex regions in the geometry of paths, Quart. J. Math. Oxford 3 (1932) 33-42. 